\title{
Ultrasonographic scan in knee pain in athletes
}

\author{
Nicola Maffulli MD, PhD, DSMSA, Renato Regine MD*, Francesco Carrillo MD*, \\ Salvatore Minelli MD*, Tina Beaconsfield BSc MB BSt \\ Department of Orthopaedics, The Hospitals for Sick Children, Great Ormond Street, London, UK, and \\ University of Naples, First Medical School, Institute of Human Physiology, Section of Sports Physiopathology, \\ Naples, Italy; *University of Naples, Second Medical School, First Institute of Radiology, Naples, Italy and \\ tDepartment of Medicine, Epsom District Hospital, Surrey, UK
}

Fifty-two knees were examined using real-time highdefinition ultrasonography with a $7.5 \mathrm{MHz}$ probe. The extra-articular structures were easily visualized and diagnosis of patellar tendon lesions and Baker's cysts formulated. While the meniscal cartilages were shown as a homogeneous triangular structure between the femoral condyle and the tibial plateau, no lesions were detected. ligaments, were not shown by the scan, thus their evaluation was not possible. Given its low cost, wide availability, non-invasiveness and patients' acceptability of the technique, ultrasonography may play an important role in the diagnosis of soft tissue lesions in and around the knee joint.

Keywords: Ultrasonography, knee injuries, sport

The knee is the most commonly injured joint in sport $^{1}$, accounting for nearly $30 \%$ of all sports injuries reported in large series ${ }^{1-4}$. Clinical diagnosis is often difficult. Conventional radiographic techniques are generally not useful in the diagnosis of soft tissue injuries, while arthrography and arthrotomography are invasive techniques with potential morbidity. Computed tomography (CT) scanning ${ }^{5}$ and magnetic resonance imaging $(\mathrm{MRI})^{6}$, while being specific, sensitive and non-invasive, are expensive, timeconsuming and available only in specialized centres.

Because of these limitations, ultrasonographic (US) scanning is useful in the diagnosis of soft tissue injuries in and around the $\mathrm{knee}^{7,8}$. It is relatively easy to perform, less expensive than CT scanning and MRI, and acceptable to patients 9 .

We report our experience of US scanning of the knee in 52 athletes.

\section{Patients and methods}

Between January 1988 and December 1989, 52 athletes with a mean(s.d.) age of 26.3(3.7) years (range 16-38 years) (Table 1) were referred for

Address for correspondence: N. Maffulli, Department of Orthopaedics, Newham General Hospital, Glen Road, Plaistow, London E13 8RU, UK Deeper intra-articular structures, such as the cruciate

diagnosis of a knee problem (Table 2). They underwent US scanning of the knee using a real-time self-focusing Aloka 500 US machine equipped with a $5 \mathrm{MHz}$ convex transducer and a $7.5 \mathrm{MHz}$ linear transducer. US scan analysis was done on photographs by a radiologist who had no prior knowledge of the patients. When necessary, commercially available soft polymer echo-free material was used as a stand-off material. This provides adequate contact between the skin and the probe, and improves image

Table 1. Sports practised

Soccer

Wrestling/judo

Track and field athletics

Rugby

Weight lifting/body building

Basketball

Others

Total
Clinical Ultrasonographic diagnosis diagnosis

\begin{tabular}{lll}
\hline & $\begin{array}{l}\text { Clinical } \\
\text { diagnosis }\end{array}$ & $\begin{array}{l}\text { Ultrasonographic } \\
\text { diagnosis }\end{array}$ \\
& & \\
\hline Baker's cyst & 6 & 9 \\
Patellar tendonitis & 9 & 5 \\
Patellar tendinosis & - & 3 \\
Patellar tendon cyst & - & 3 \\
Partial rupture of patellar tendon & 1 & 2 \\
Quadriceps tendonitis & 6 & - \\
Meniscal pathology and/or & & - \\
$\quad$ ligamentous lesions & 9 & 1 \\
Pericondylar calcification & - & 7 \\
Fat pad syndrome & 5 & - \\
Infrapatellar bursitis & 2 & 7 \\
Lateral meniscal cyst & 7 & (see Baker's \\
Posterior knee pain & 3 & cyst) \\
& & (see patellar \\
Anterior knee pain & 4 & tendon and \\
& & calcification) \\
& &
\end{tabular}

Table 2. Comparison between clinical and ultrasonographic diagnosis 
quality by placing the various structures in the optimal focal zone of the transducer ${ }^{10}$. US studies were carried out with the knee flexed at various angles to gain a dynamic image of the structures examined. Longitudinal, transverse and oblique scans were used. The contralateral knee was always examined for comparison. The probe was placed parallel to the major axis of the tendons during longitudinal scans and perpendicular to it during transverse scans, so as not to create false echoic patterns $^{10}$.

\section{Results}

Only a few acutely injured knees were examined, thus the series presented is biased towards chronic and overuse conditions. The results of clinical and ultrasonographic examination are shown in Table 2.

\section{Baker's cysts}

Nine patients presented with pain and swelling in the popliteal fossa. US scanning revealed an ovoid cystic space filled with fluid (Figure 1). The cysts appeared as a hypoechoic fluid collection in the popliteal fossa, at various depths from the skin. In no cases were ruptures and/or soft tissue changes resulting from a recent leak identified ${ }^{11}$.

\section{Tendons}

Patellar tendon

Patellar tendon injury is common ${ }^{12}$. Transverse US scanning did not give useful information. In longitudinal scans, the normal patellar tendon appeared as a 4-5 mm thick ribbon composed of parallel hypo- and hyperechoic zones alternating regularly in waveform, and with well-defined slightly hyperechoic borders (Figure 2). At its insertion into the anterior tibial tubercle, the patellar tendon was hypoechoic and

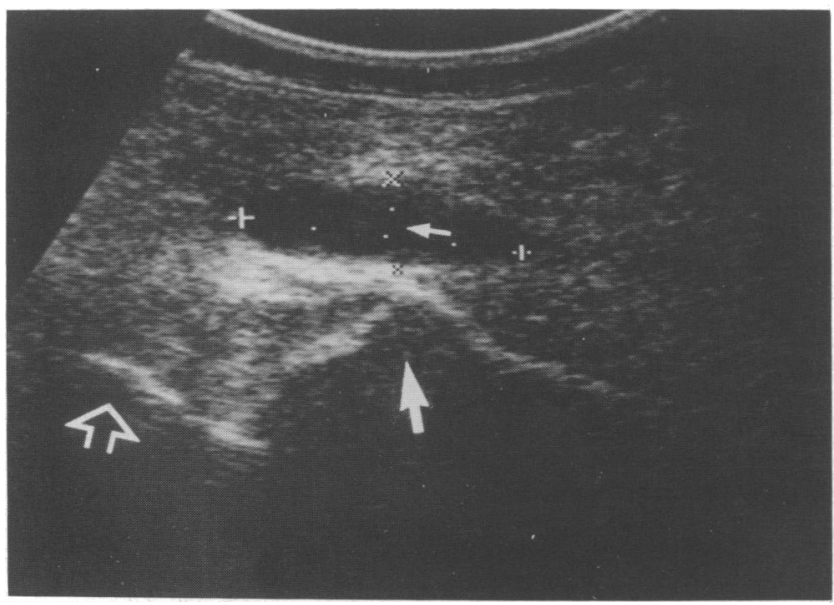

Figure 1. Longitudinal scan of the posterior aspect of the knee. The posterior aspect of the femur (empty arrow) and the tibia (large solid arrow) give a hyperechoic signal with acoustic shadowing. A Baker's cyst (small arrow) appears as an ovoid hyperechoic space (delimited by the ' + ' and the ' $(x$ '), with no evidence of communication with the knee joint slightly enlarged 7 . In eight cases, diffuse thickening was evident, involving either the entire tendon or a segment. Swelling was present in four cases, and some areas of reduced attenuation or decreased echogenicity were identified, probably representing granulation tissue or oedema ${ }^{13}$. These findings were compatible with a diagnosis of patellar tendonitis and tendinosis, respectively ${ }^{13}$.

In three cases, a well defined unilocular cyst in the substance of the patellar tendon was shown, together with thickening of the tendon and swelling of its lining (Figure 3). Finally, in two other cases a large

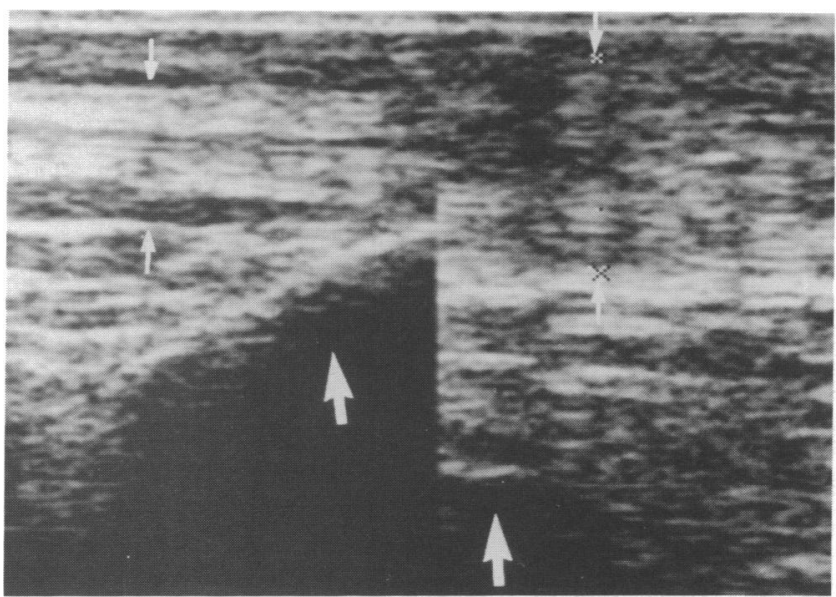

Figure 2. Bilateral longitudinal scan of the patellar tendon. The large arrows are placed in the tibia. The small arrows point to the patellar tendon. The normal tendon, on the left-hand side, appears as a ribbon $4-5 \mathrm{~mm}$ thick, with parallel hypoechoic and hyperechoic areas alternating in a regular fashion. The borders are slightly hyperechoic, and in the area of tendinous insertion the tendon is hypoechoic and enlarged. On the right-hand side, the tendon shows diffuse thickening, with loss of the normal regular waveform structure

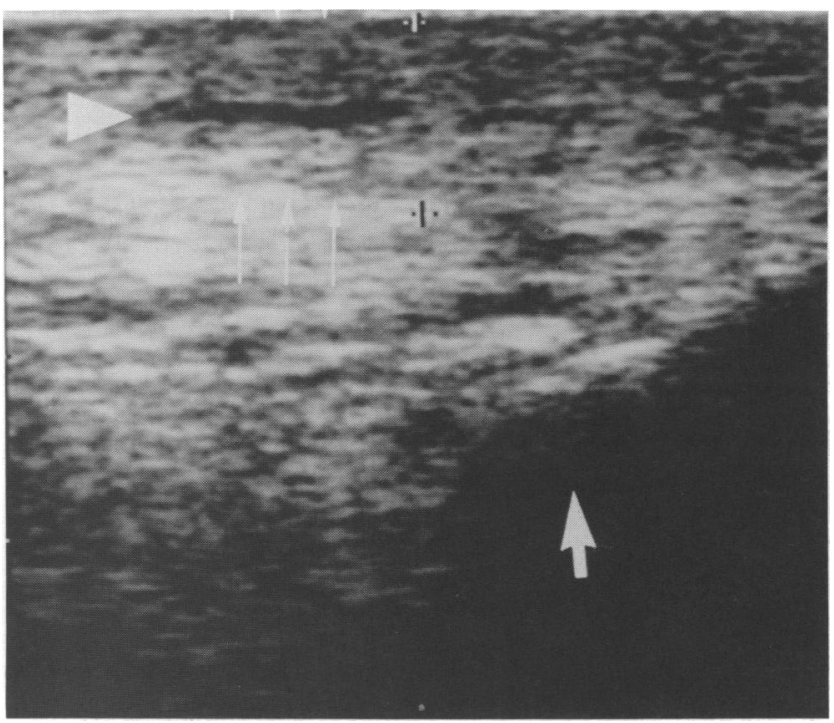

Figure 3. Patellar tendon cyst, longitudinal scan. The large arrow is in the tibia, the small arrows point to the anterior and posterior wall of the patellar tendon, which is enlarged. A triangle points at the superior pole of a hypoechoic tendon cyst 
cystic space was present, with marked loss of the normal waveform structure of the tendon. Some fine hyperechoic areas were identified (Figure 4). US diagnosis of partial rupture of the patellar tendon was confirmed at operation.

\section{Quadriceps tendon}

Six patients were referred with knee pain above the superior pole of the patella. The quadriceps tendon was examined and found to be of the same thickness (about $3 \mathrm{~mm}$ ) as the contralateral (Figure 5). The usual tendinous waveform pattern was found and a clinical diagnosis of 'quadriceps tenalgia' formulated.

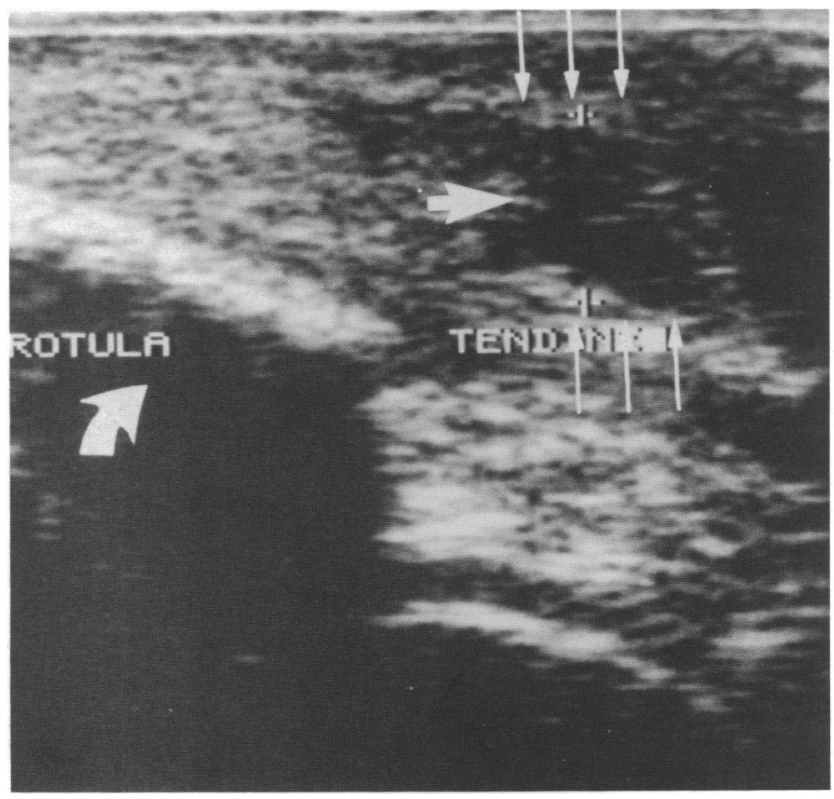

Figure 4. Partial rupture of the patellar tendon, oblique view. The large arrow is in the tibia and the curved arrow is in the patella. The small arrows point to the anterior and posterior wall of the patellar tendon, which is enlarged proximally, oedematous, with partial destruction of its substance. The straight arrows point at a vast cystic area, surgically an intratendinous haematoma.

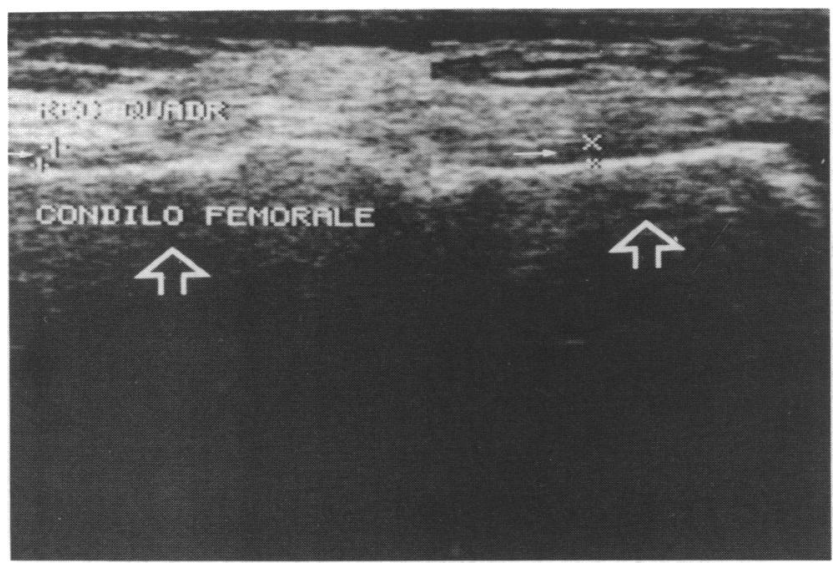

Figure 5. Normal quadriceps tendon. On the left- and right-hand side, the empty arrow is placed in the substance of the femur. The quadriceps tendon (small arrows) is ribbon-like, with no evidence of pathology

\section{Fat pad syndrome}

In seven athletes, a bursa was excluded on the basis of diffuse echogenicity alteration of the fat pad, normally appearing as an area of homogeneous hyperechogenicity behind the tendon (Figure 6). In two cases, a diagnosis of deep infrapatellar bursitis had been clinically suggested. No US signs of bursal involvement were detected, and a bursitis was thus excluded, the findings being consistent with fat pad syndrome.

\section{Menisci and ligaments}

In four athletes referred for pain and tenderness in the medial joint line, the menisci were easily visualized. They appeared as a homogeneous triangular structure, with the apex of the triangle pointing towards the middle of the joint ${ }^{14}$. The meniscal attachment to the capsular ligaments was well seen, without an echoic interface. The posterior horn of the medial meniscus was always easily visualized, and no pathological appearances could be detected (Figure 7).

The anterior and posterior cruciate ligaments were poorly, if at all, visualized ${ }^{15}$, and it was not possible to formulate a definite diagnosis on their integrity.

\section{Meniscal cysts}

Seven athletes presented with a swelling on the lateral aspect of the knee, clinically a cyst of the lateral meniscus. US scanning revealed an hypoechoic lesion with several fibrous septa, illustrating the multiloculation of meniscal cysts as previously shown on CT scans ${ }^{16}$.

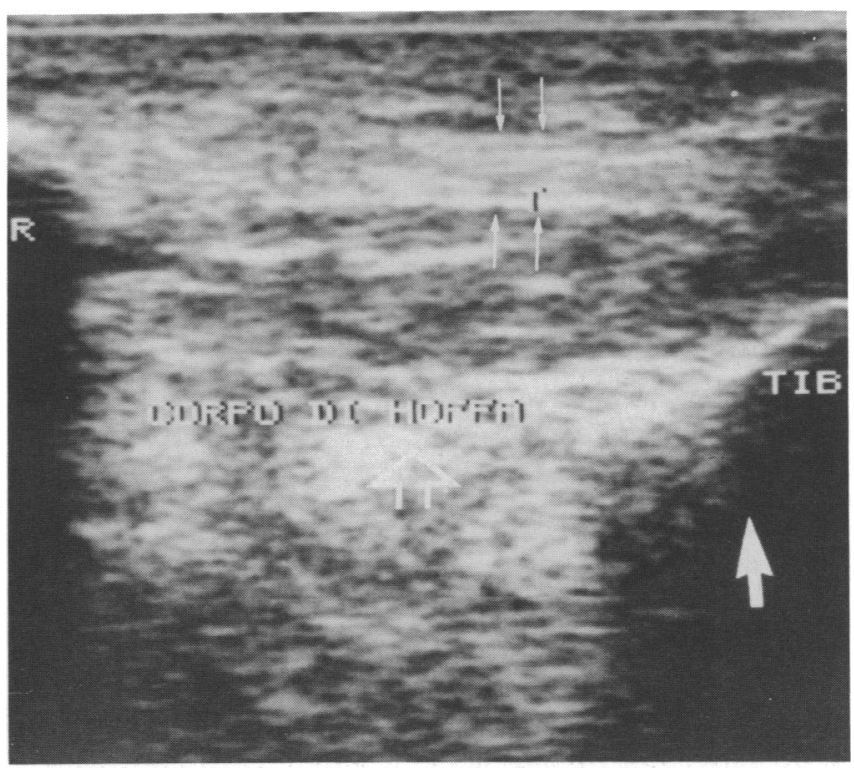

Figure 6. Fat pad syndrome. The large arrow is placed in the tibia, the small arrows point to the anterior and posterior wall of the patellar tendon, and the empty arrow is in the fat pad. This appears as a dishomogeneous formation with several hypoechoic areas 


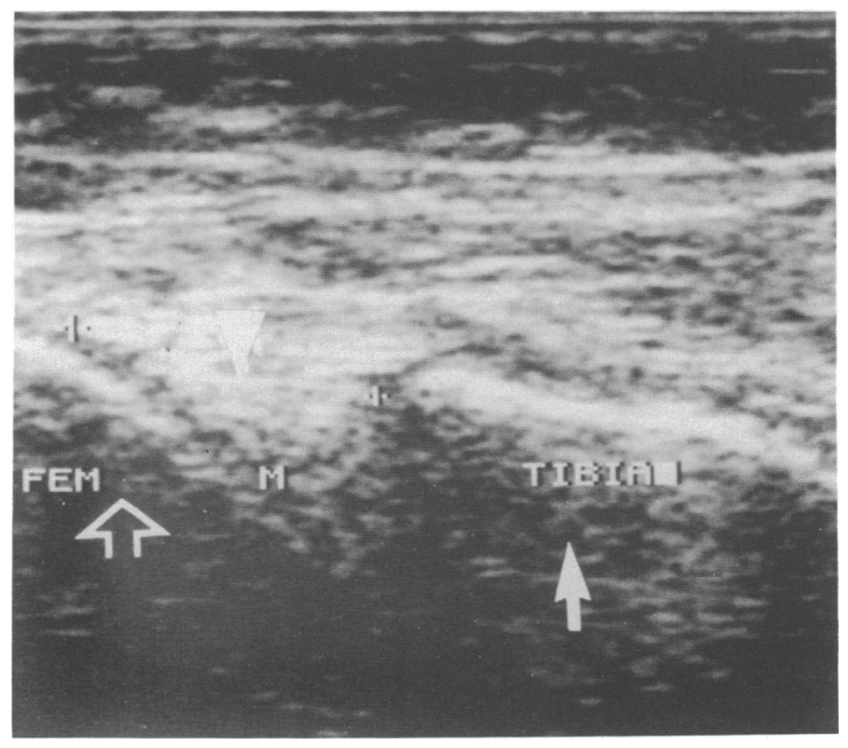

Figure 7. Posterior horn of the medial meniscus. The empty arrow is placed in the femur, the other in the tibia. The meniscus (triangle) is normal, and is visualized as a homogeneous triangular structure, with the apex of the triangle pointing towards the inner side of the joint

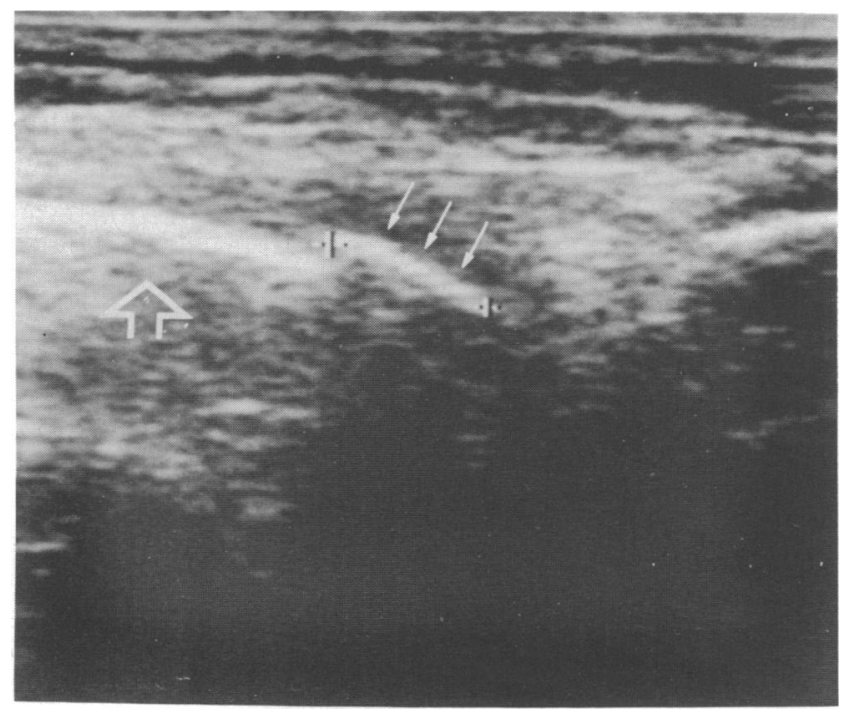

Figure 8. Pericondylar calcification. A smooth, linear hyperechoic formation (small arrows) is seen above the femoral condyle (empty arrow), normally appearing as a rounded formation (see other figures)

\section{Articular surfaces}

The bony surfaces of the femoral condyles and the tibial plateau gave a highly hyperechoic signal, with acoustic shadowing. The hypoechoic rim of the articular cartilage surrounded the femoral condyles. In one case, pericondylar calcification was shown (Figure 8). It appeared as a smooth, linear hyperechoic formation, without the rounded profile of the normal femoral condyle.

In 15 patients, no abnormalities were detected.

\section{Discussion}

US is a useful diagnostic technique in the painful knee. Baker's cyst, popliteal artery aneurysms and neural tumours may all present as painful extraarticular masses in the popliteal fossa. US is a useful method of differentiating between them.

Although we did not find any meniscal lesions, the medial meniscus was easily shown on US scans, and, in the light of recent experience ${ }^{8}$, the sensitivity and specificity of US should allow the diagnosis of tears. The cruciate ligaments are difficult to demonstrate because they lie deep in the joint. This difficulty may be partly due to the nature of the injuries seen. A meniscal or cruciate lesion generally results in an 'acute knee', while this series is strongly biased towards chronic or overuse injuries.

More work comparing the clinical and US findings needs to be performed, to accumulate data on knee conditions and lesions which may benefit from diagnostic US scanning. With further technical refinements, an expansion of the role of US in the evaluation of the knee is anticipated.

\section{References}

1 Kujala UM, Kvist M, Osterman K. Knee injuries in athletes. Review of exertion injuries and retrospective study of outpatient sports clinic material. Sports Med 1986; 3: 447-60.

2 Kvist M, Jarvinen M. Zur Epidemiologie von sportverletzungen und Fehlbelastungsfolen: Patienten-analyse einer sportsmedizinischen Poliklinik. Med Sport 1980; 20: 375-8.

3 Newman PH, Thomson JPS, Barnes JM, Moore TMC. A clinic for athletic injuries. Proc $R$ Soc Med 1969; 62: 939-44.

4 Orava S, Puranen J. Athlete exertion injuries. Ann Chir Gynaecol 1978; 67: 58-65.

5 Rosemberg ZG, Feldman F, Singson RD. Peroneal tendon injuries: CT analysis. Radiology 1986; 161: 743-50.

6 Ehman RL, Berquist TH. Magnetic resonance imaging of musculoskeletal trauma. Radiol Clin North Am 1986; 24: 291-7.

7 Laine HR, Harijula A, Peltokallio P. Ultrasound evaluation of the knee and patellar regions. I Ultrasound Med 1987; 6: 33-6.

8 Casser H-R, Sohn C, Kiekenbeck A. Current evaluation of sonography of the meniscus. Results of a comparative study of sonographic and arthroscopic findings. Arch Orthop Trauma Surg 1990; 109: 150-4.

9 Harcke HT, Grisson LE, Finklestein MS. Evaluation of the musculoskeletal system with sonography. AJR 1988; 150: 1253-61.

10 Fornage BD. The hypoechoic normal tendon. A pitfall. J Ultrasound Med 1987; 6: 19-22.

11 Gompels BM, Darlington LG. Evaluation of popliteal cysts and painful calves with ultrasonography. Comparison with arthrography. Ann Rheum Dis 1982; 41: 355-9.

12 Fritschy D, de Gautard R. Jumper's knee and ultrasonography. Am J Sports Med 1988; 16: 637-40.

13 King JB, Perry DJ, Mourad K, Kumar SJ. Lesions of the patellar ligament. J Bone Joint Surg [Br] 1990; 72-B: 46-8.

14 Selby B, Richardson ML, Montana $M$ et al. High resolution sonography of the menisci of the knee. Invest Radiol 1986; 21: 332-5.

15 Richardson ML, Selby B, Montana M, Mack LA. Ultrasonography of the knee. Radiol Clin North Am 1988; 26: 63-75.

16 Chen W-C, Wu JJ, Chang C-Y et al. Computed tomography of a meniscal cyst. Orthopedics 1987; 10: 1569-72. 